PROCEEDINGS OF THE

AMERICAN MATHEMATICAL SOCIETY

Volume 140, Number 7, July 2012, Pages 2447-2451

S 0002-9939(2011)11288-7

Article electronically published on November 21, 2011

\title{
NONLINEAR PERTURBATIONS OF LINEAR ELLIPTIC SYSTEMS AT RESONANCE
}

\author{
PHILIP KORMAN
}

(Communicated by Walter Craig)

AbStract. We consider a semilinear system

$$
\begin{array}{lll}
\Delta u+\lambda v+b_{1}(v)=f(x), \quad x \in \Omega, & u=0 & \text { for } x \in \partial \Omega, \\
\Delta v+\frac{\lambda_{1}^{2}}{\lambda} u+b_{2}(u)=g(x), \quad x \in \Omega, & v=0 & \text { for } x \in \partial \Omega,
\end{array}
$$

whose linear part is at resonance. Here $\lambda>0$ and the functions $b_{1}(t)$ and $b_{2}(t)$ are bounded and continuous. Assuming that $t b_{i}(t)>0$ for all $t \in R, i=1,2$, and that the first harmonics of $f(x)$ and $g(x)$ lie on a certain straight line, we prove the existence of solutions. This extends a similar result for one equation, due to D.G. de Figueiredo and W.-M. Ni.

\section{INTRODUCTION}

Following the publication of the classical paper of E.M. Landesman and A.C. Lazer [7, there has been enormous interest in nonlinear perturbations of linear equations at resonance of the type

$$
\Delta u+\lambda_{1} u+b(u)=f(x), \quad x \in \Omega, u=0 \text { for } x \in \partial \Omega,
$$

where $\Omega$ is a bounded smooth domain in $R^{n}$ and $\lambda_{1}$ is the principal eigenvalue of the Laplacian $-\Delta$ on $\Omega$, with zero at the boundary condition (we shall denote by $\phi_{1}(x)$ the corresponding eigenfunction). Early contributions included the other classics, such as A. Ambrosetti and G. Prodi 1 and M.S. Berger and E. Podolak 3]; see a nice presentation in the book of A. Ambrosetti and G. Prodi 22. Recently, the present author [ $[$ has suggested a unified approach to these results. The function $b(u)$ is usually assumed to be bounded and continuous, and the famous E.M. Landesman and A.C. Lazer [7] conditions required that it had limits at $\pm \infty$. In an elegant paper, D.G. de Figueiredo and W.-M. Ni 5 proved the existence of solutions assuming that $u b(u)>0$ for all $u \in R$ and the forcing term $f(x)$ has zero first harmonic; i.e., $\int_{\Omega} f(x) \phi_{1}(x) d x=0$. Their proof involved the establishment of an a priori estimate, which was remarkable because such estimates usually require some conditions on $b(u)$ at infinity.

In this paper we extend the result of D.G. de Figueiredo and W.-M. Ni 5 to a system of two equations. The system

$$
\begin{gathered}
\Delta u+\lambda v+b_{1}(v)=f(x), \quad x \in \Omega, \quad u=0 \text { for } x \in \partial \Omega, \\
\Delta v+\frac{\lambda_{1}^{2}}{\lambda} u+b_{2}(u)=g(x), \quad x \in \Omega, \quad v=0 \text { for } x \in \partial \Omega,
\end{gathered}
$$

Received by the editors February 25, 2011.

2010 Mathematics Subject Classification. Primary 35J60.

Key words and phrases. Elliptic system at resonance, existence of solutions. 
with any $\lambda>0$ can be seen as the case of resonance at the principal eigenvalue, similar to (1.1). Similarly to [5], we assume that $t b_{i}(t)>0$ for all $t \in R, i=1,2$. We prove the existence of solutions, provided that the first harmonics of $f(x)$ and $g(x)$ lie on a certain straight line. There is considerable interest in systems of this type; see, e.g., the recent surveys of D.G. de Figueiredo [4] and B. Ruf [9].

\section{Existence of SOlutions}

On a smooth domain $\Omega \subset R^{n}$, we consider a weakly coupled linear system

$$
\begin{array}{ll}
\Delta u+\lambda v=f(x), & x \in \Omega, \quad u=0 \text { for } x \in \partial \Omega, \\
\Delta v+\bar{\lambda} u=g(x), \quad x \in \Omega, \quad v=0 \text { for } x \in \partial \Omega,
\end{array}
$$

with given functions $f(x)$ and $g(x)$, and parameters $\lambda$ and $\bar{\lambda}$. The following proposition identifies the set of nonresonant parameters $\lambda$ and $\bar{\lambda}$. We denote by $\lambda_{n}$ the eigenvalues of $-\Delta$ on $\Omega$, with zero boundary condition, and by $\phi_{n}(x)$ the corresponding eigenfunctions.

Proposition 1. Assume that $\lambda \bar{\lambda} \neq \lambda_{n}^{2}$ for all $n \geq 1$. Then for any pair $(f(x), g(x))$ $\in L^{2}(\Omega) \times L^{2}(\Omega)$ there exists a unique solution $(u(x), v(x)) \in\left(W^{2,2}(\Omega) \cap W_{0}^{1,2}(\Omega)\right)^{2}$.

Proof. Existence of a solution in $L^{2}(\Omega) \times L^{2}(\Omega)$ follows by using the Fourier series in $\phi_{n}(x)$, written for $u, v, f$, and $g$, and then the standard elliptic estimates provide the extra regularity of a solution.

The resonance case is when $\lambda \bar{\lambda}=\lambda_{n}^{2}$. We shall consider the principal resonance case $\lambda \bar{\lambda}=\lambda_{1}^{2}$, i.e., $\bar{\lambda}=\frac{\lambda_{1}^{2}}{\lambda}$. We shall prove solvability for the system

$$
\begin{aligned}
& \Delta u+\lambda v+b_{1}(v)=f(x), \quad x \in \Omega, \quad u=0 \text { for } x \in \partial \Omega, \\
& \Delta v+\frac{\lambda_{1}^{2}}{\lambda} u+b_{2}(u)=g(x), \quad x \in \Omega, \quad v=0 \text { for } x \in \partial \Omega,
\end{aligned}
$$

with given functions $f(x), g(x) \in L^{2}(\Omega)$, and a constant $\lambda>0$. The following is a system analog of the result of D.G. de Figueiredo and W.-M. Ni [5]. We denote $\phi_{1}^{\perp}=\left\{f \in L^{2}(\Omega): \int_{\Omega} f \phi_{1} d x=0\right\}$.

Theorem 2.1. Assume that $b_{1}(t)$ and $b_{2}(t)$ are bounded and continuous functions such that

$$
t b_{i}(t)>0 \text { for all } t \in R, i=1,2 \text {. }
$$

Decompose $f(x)=\mu_{1} \phi_{1}(x)+e_{1}(x), g(x)=\nu_{1} \phi_{1}(x)+e_{2}(x)$, with $e_{1}(x), e_{2}(x) \in \phi_{1}^{\perp}$. Then the system (2.2) is solvable for any $\left(\mu_{1}, \nu_{1}\right)$ satisfying

$$
\lambda_{1} \mu_{1}+\lambda \nu_{1}=0
$$

with $u, v \in W_{0}^{1,2}(\Omega) \cap W^{2, p}(\Omega)$, for all $p>2$.

The proof will be based on the following lemmas. The first one follows immediately by considering the Fourier series in $\phi_{n}(x)$.

Lemma 2.1. The solution set of the linear system

$$
\begin{aligned}
& \Delta u+\lambda v=0, \quad x \in \Omega, \quad u=0 \text { for } x \in \partial \Omega, \\
& \Delta v+\frac{\lambda_{1}^{2}}{\lambda} u=0, \quad x \in \Omega, \quad v=0 \text { for } x \in \partial \Omega,
\end{aligned}
$$

is $(u, v)=c\left(\phi_{1}, \frac{\lambda_{1}}{\lambda} \phi_{1}\right)$, where $c$ is an arbitrary constant. In particular, the only solution of (2.5) in $\phi_{1}^{\perp} \times \phi_{1}^{\perp}$ is $(0,0)$. 
Lemma 2.2. Let $U, V \in \phi_{1}^{\perp}$ be solutions of

$$
\begin{gathered}
\Delta U+\lambda V=f(x), \quad x \in \Omega, \quad u=0 \text { for } x \in \partial \Omega, \\
\Delta V+\frac{\lambda_{1}^{2}}{\lambda} U=g(x), \quad x \in \Omega, \quad v=0 \text { for } x \in \partial \Omega,
\end{gathered}
$$

with $f(x), g(x) \in L^{\infty}(\Omega)$. Then for any $p>1$ one can find a constant $c>0$ such that

$$
\|U\|_{W^{2, p}(\Omega)}+\|V\|_{W^{2, p}(\Omega)} \leq c\left(\|f\|_{L^{p}(\Omega)}+\|g\|_{L^{p}(\Omega)}\right) .
$$

By the Sobolev imbedding this implies that $\|U\|_{L^{\infty}(\Omega)}+\|V\|_{L^{\infty}(\Omega)} \leq c_{1}$ for some constant $c_{1}>0$.

Proof. Standard elliptic estimates imply that

$$
\|U\|_{W^{2, p}}+\|V\|_{W^{2, p}} \leq c\left(\|f\|_{L^{p}}+\|g\|_{L^{p}}+\|U\|_{L^{p}}+\|V\|_{L^{p}}\right) .
$$

The estimate (2.7) will follow, once we prove that

$$
\|U\|_{L^{p}}+\|V\|_{L^{p}} \leq c\left(\|f\|_{L^{p}}+\|g\|_{L^{p}}\right) .
$$

Assume for definiteness that $\|f\|_{L^{p}} \geq\|g\|_{L^{p}}$. Dividing both equations in (2.7) by the same constant $\|f\|_{L^{p}}$ and redefining $U$ and $V$, we may assume that $\|f\|_{L^{p}}=1$ and $\mid g \|_{L^{p}} \leq 1$. Assuming that the estimate (2.8) is not possible with any constant $c$, we could find a sequence $\left\{f_{n}, g_{n}\right\}$, with $\left\|f_{n}\right\|_{L^{p}}=1$ and $\mid g_{n} \|_{L^{p}} \leq 1$, and the corresponding solutions of (2.6) $\left\{U_{n}, V_{n}\right\} \in \phi_{1}^{\perp} \times \phi_{1}^{\perp}$ so that

$$
\left\|U_{n}\right\|_{L^{p}}+\left\|V_{n}\right\|_{L^{p}} \geq n\left(1+\left\|g_{n}\right\|_{L^{p}}\right) .
$$

In particular, $\left\|U_{n}\right\|_{L^{p}}+\left\|V_{n}\right\|_{L^{p}} \rightarrow \infty$, as $n \rightarrow \infty$. Define $u_{n}=\frac{U_{n}}{\left\|U_{n}\right\|_{L^{p}}+\left\|V_{n}\right\|_{L^{p}}}$ and $v_{n}=\frac{V_{n}}{\left\|U_{n}\right\|_{L^{p}}+\left\|V_{n}\right\|_{L^{p}}}$. They satisfy

$$
\begin{aligned}
\Delta u_{n}+\lambda v_{n} & =\frac{f_{n}(x)}{\left\|U_{n}\right\|_{L^{p}}+\left\|V_{n}\right\|_{L^{p}}}, \\
\Delta v_{n}+\frac{\lambda_{1}^{2}}{\lambda} u_{n} & =\frac{g_{n}(x)}{\left\|U_{n}\right\|_{L^{p}}+\left\|V_{n}\right\|_{L^{p}}} .
\end{aligned}
$$

Since $\left\|u_{n}\right\|_{L^{p}}<1,\left\|v_{n}\right\|_{L^{p}}<1$, we get uniform in $n$ bounds for $\left\|u_{n}\right\|_{W^{2, p}}$ and $\left\|v_{n}\right\|_{W^{2, p}}$. In a standard way, along a subsequence $\left\{u_{n}, v_{n}\right\} \rightarrow(u, v) \in \phi_{1}^{\perp} \times \phi_{1}^{\perp}$, with $(u, v)$ solving (2.5). Hence $u=v=0$ by Lemma 2.2, but $\|u+v\|_{L^{p}}=1$, a contradiction.

The following lemma provides the crucial a priori estimate. As mentioned by D.G. de Figueiredo and W.-M. Ni, it is remarkable that this estimate does not require any conditions on $b_{i}(t)$ at infinity (which are usually needed to get a priori estimates).

Lemma 2.3. In the conditions of the Theorem 2.1, there is a constant $c>0$ so that any solution of (2.2) satisfies

$$
\|u\|_{L^{2}(\Omega)}+\|v\|_{L^{2}(\Omega)} \leq c .
$$

Proof. Decompose $u(x)=\xi_{1} \phi_{1}(x)+U(x), v(x)=\eta_{1} \phi_{1}(x)+V(x)$, with $U(x)$, $V(x) \in \phi_{1}^{\perp}$. Then system (2.2) becomes

$$
\begin{aligned}
& \Delta U+\lambda V+\left(-\lambda_{1} \xi_{1}+\lambda \eta_{1}\right) \phi_{1}+b_{1}\left(\eta_{1} \phi_{1}(x)+V(x)\right)=\mu_{1} \phi_{1}(x)+e_{1}(x), \\
& \Delta U+\frac{\lambda_{1}^{2}}{\lambda} U+\frac{\lambda_{1}}{\lambda}\left(\lambda_{1} \xi_{1}-\lambda \eta_{1}\right) \phi_{1}+b_{2}\left(\xi_{1} \phi_{1}(x)+U(x)\right)=\nu_{1} \phi_{1}(x)+e_{2}(x) .
\end{aligned}
$$


We claim that

$$
\left|-\lambda_{1} \xi_{1}+\lambda \eta_{1}\right| \leq c
$$

for some constant $c>0$. Indeed, multiply the first equation in (2.10) by $\phi_{1}$ and integrate over $\Omega$. Since $\int_{\Omega} \Delta U \phi_{1} d x=\int_{\Omega} V \phi_{1} d x=0$, while $b_{1}$ is a bounded function, the claim follows. By Lemma 2.2, it follows that

$$
\|U\|_{C^{1}(\Omega)}+\|V\|_{C^{1}(\Omega)} \leq c_{1}
$$

for some constant $c_{1}>0$.

To complete the proof, we need an a priori estimate of the first harmonics $\xi_{1}$ and $\eta_{1}$. By (2.11), if either one of $\xi_{1}$ and $\eta_{1}$ is large and positive (negative), so is the other one. Assume for definiteness that $\xi_{1}$ and $\eta_{1}$ are both negative and large in absolute value. Multiply the first equation in (2.10) by $\lambda_{1} \phi_{1}$, the second one by $\lambda \phi_{1}$, integrate over $\Omega$, and add the results. We may assume that $\int_{\Omega} \phi_{1}^{2} d x=1$. By our condition (2.4)

$$
0=\lambda_{1} \mu_{1}+\lambda \nu_{1}=\int_{\Omega}\left[\lambda_{1} b_{1}\left(\eta_{1} \phi_{1}(x)+V(x)\right)+\lambda b_{2}\left(\xi_{1} \phi_{1}(x)+U(x)\right)\right] \phi_{1}(x) d x .
$$

We claim that the integral on the right is negative, which gives us a contradiction. Indeed, by (2.12), $\eta_{1} \phi_{1}(x)+V(x)<0$ and $\xi_{1} \phi_{1}(x)+U(x)<0$ over $\Omega$, and then, by condition (2.3), the functions $b_{1}$ and $b_{2}$ are negative.

Proof of the Theorem 2.1. Letting $w=(u, v)$, we rewrite the system (2.2) in the operator form

$$
w=T(w),
$$

where $T(w)=\left(\Delta^{-1}\left(-\lambda v-b_{1}(v)+\mu_{1} \phi_{1}+e_{1}\right), \Delta^{-1}\left(-\frac{\lambda_{1}^{2}}{\lambda} u-b_{2}(u)+\nu_{1} \phi_{1}+e_{2}\right)\right)$. $T$ is a compact map $L^{2}(\Omega) \times L^{2}(\Omega) \rightarrow L^{2}(\Omega) \times L^{2}(\Omega)$. We define $\mathbf{L}^{2}=L^{2}(\Omega) \times$ $L^{2}(\Omega)$, with the norm $\|w\|_{\mathbf{L}^{2}}^{2}=\|u\|_{L^{2}(\Omega)}^{2}+\|v\|_{L^{2}(\Omega)}^{2}$. Following D.G. de Figueiredo and W.-M. Ni [5], we consider the operator

$$
T_{k}(w)=\frac{1}{k+1} T(w)-\frac{k}{k+1} T(-w), \quad 0 \leq k \leq 1,
$$

which is compact for all $k, T_{0}=T$, and $T_{1}$ is an odd operator. It is known (see, e.g., L. Nirenberg [8]) that the Leray-Schauder degree

$$
\operatorname{deg}\left(I-T_{1}, B_{R}, 0\right) \neq 0
$$

for any ball $B_{R}=\left\{w \in \mathbf{L}^{2}:\|w\|_{\mathbf{L}^{2}} \leq R\right\}$. We claim that there is an $R$ such that

$$
w-T_{k}(w) \neq 0, \text { for }\|w\|_{\mathbf{L}^{2}}=R, \quad 0 \leq k \leq 1 .
$$

Then by the homotopy invariance of the degree, $\operatorname{deg}\left(I-T, B_{R}, 0\right) \neq 0$, which implies that system (2.2) has a solution. To prove the claim, we need a uniform in $k$ a priori bound for

$$
w-T_{k}(w)=0,
$$

which is equivalent to

$$
\begin{aligned}
& \Delta u+\lambda v+\frac{1}{k+1} b_{1}(v)-\frac{k}{k+1} b_{1}(-v)=\frac{1-k}{1+k}\left(\mu_{1} \phi_{1}+e_{1}\right), \\
& \Delta v+\frac{\lambda_{1}^{2}}{\lambda} u+\frac{1}{k+1} b_{2}(u)-\frac{k}{k+1} b_{2}(-u)=\frac{1-k}{1+k}\left(\nu_{1} \phi_{1}+e_{2}\right) .
\end{aligned}
$$

Clearly, the condition (2.4) on the first harmonics is satisfied for all $k$. Letting $b_{i}^{k}(t)=\frac{1}{k+1} b_{i}(t)-\frac{k}{k+1} b_{i}(-t), i=1,2$, we see that these functions are uniformly 
bounded in $k$ and satisfy the condition (2.3). By Lemma2.3 we conclude a uniform in $k$ a priori bound for solutions of (2.13), completing the proof.

\section{ACKNOWLEDGMENT}

It is a pleasure to thank Wei-Ming Ni, who explained [5] to the author at about the time of its publication in 1979. It did take the author a while to absorb that information.

\section{REFERENCES}

[1] A. Ambrosetti and G. Prodi, On the inversion of some differentiable mappings with singularities between Banach spaces, Ann. Mat. Pura Appl. 93 (4), 231-246 (1972). MR0320844 (47:9377)

[2] A. Ambrosetti and G. Prodi, A Primer of Nonlinear Analysis. Cambridge Studies in Advanced Mathematics, 34. Cambridge University Press, Cambridge (1993). MR1225101 (94f:58016)

[3] M.S. Berger and E. Podolak, On the solutions of a nonlinear Dirichlet problem, Indiana Univ. Math. J. 24, 837-846 (1974/75). MR0377274(51:13447)

[4] D.G. de Figueiredo, Semilinear elliptic systems: Existence, multiplicity, symmetry of solutions, Handbook of Differential Equations, Stationary Partial Differential Equations, Vol. 5, edited by M. Chipot, Elsevier Science, North-Holland, 1-48 (2008). MR2497896|(2010j:35126)

[5] D.G. de Figueiredo and W.-M. Ni, Perturbations of second order linear elliptic problems by nonlinearities without Landesman-Lazer condition, Nonlinear Anal. 3, no. 5, 629-634 (1979). MR541873(81k:35052)

[6] P. Korman, Curves of equiharmonic solutions, and ranges of nonlinear equations, Adv. Differential Equations 14, no. 9-10, 963-984 (2009). MR2548284 (2010m:35164)

[7] E.M. Landesman and A.C. Lazer, Nonlinear perturbations of linear elliptic boundary value problems at resonance, J. Math. Mech. 19, 609-623 (1970). MR0267269 (42:2171)

[8] L. Nirenberg, Topics in Nonlinear Functional Analysis, Courant Institute Lecture Notes, Amer. Math. Soc. (1974). MR.1850453(2002j:47085)

[9] B. Ruf, Superlinear elliptic equations and systems, Handbook of Differential Equations, Stationary Partial Differential Equations, Vol. 5, edited by M. Chipot, Elsevier Science, NorthHolland, 277-370 (2008). MR2497908 (2010d:35107)

Department of Mathematical Sciences, University of Cincinnati, Cincinnati, Ohio 45221-0025

E-mail address: kormanp@math.uc.edu 